\title{
Embedded System Interfacing with GNSS user Receiver for Transport Applications
}

\author{
Mohmad Umair Bagali ${ }^{1}$ \\ Research Scholar \\ Department of Electronics and Communication Engineering \\ JAIN (Deemed-to-be University), Bangalore, India
}

\author{
Dr. Thangadurai. $\mathrm{N}^{2}$ \\ Professor and Research Coordinator \\ Department of Electronics and Communication Engineering \\ JAIN (Deemed-to-be University), Bangalore, India
}

\begin{abstract}
The real time vehicle movement traces using waypoint display on the base-map with IRNSS/NavIC and GPS dataset in the GUI simultaneously. In this paper, a portable electronic device with application software has been designed and developed, which would be used to capture the real time positional information of a rover using IRNSS-UR. It stores the positional information into database and displays the real time vehicle positional information like date, time, latitude, longitude and altitude using both GPS and IRNSS/NavIC receiver simultaneously. The designed hardware device with an application software developed helps in mapping the real time vehicle / rover movement at the same time which also helps in identifying the region with data loss, varying positional information, comparing the distance travelled by rover and also aid in retrieving the past surveys and mapping the traces of both IRNSS and GPS simultaneously. The vehicle movement using both IRNSS/NavIC and GPS are tracked on the base map to find the similarity and differences between two. During this research work it can be conclude that that the rover position using GPS and IRNSS were accurate and continuous in our survey duration except in few places. In that few places the data loss is observed because of the satellite visibility variations. For Indian region the IRNSS/NavIC can be a better replacement for GPS.
\end{abstract}

\section{Keywords-GNSS; GPS; IRNSS; embedded systems}

\section{INTRODUCTION}

NavIC/IRNSS is a free provincial regional satellite constellation being developed by India. IRNSS will give two kinds of administration services, to be specific, Standard Positioning Service (SPS) which is given to every one of the customers and Restricted Service (RS), which is an encoded administration given distinctly to the approved clients only. It is intended to give precise position data administration to customers in India just as the locale extending out up to 1500 $\mathrm{km}$ from its limit, which is its essential administration region. An all-inclusive administration territory lies between essential administration region from Latitude $30^{\circ} \mathrm{S}$ to $50^{\circ} \mathrm{N}$ and Longitude 30 degree East to 130 degree East [2]. The IRNSS system is relied upon to give a position precision of superior to $10 \mathrm{~m}$ in the essential administration region [5].

The NavIC system comprises a ground segment which is supported by a space segment.

Space segment: 8 satellites constellation, where 4 satellites are in Geosynchronous Orbit (GSO) crossing the equator at $55^{\circ}$ East and two at $111.75^{\circ}$ East and three satellites are located approximately $36,000 \mathrm{~km}(22,000 \mathrm{mi})$ above earth surface in Geostationary Orbit (GEO) at $83^{\circ}$ East, $32.5^{\circ}$ East, and $131.5^{\circ}$ East longitude. The movement of four GSO satellites will be in the form of figure of " 8 " as shown in Fig. 1.

Ground segment: The maintenance and operation of the IRNSS constellation is held in ground segment (Fig. 2) are:

1) IRNSS Spacecraft Control Facility (IRSCF)

2) ISRO Navigation Centre (INC)

3) IRNSS Range and Integrity Monitoring Stations (IRIMS)

4) IRNSS Network Timing Centre (IRNWT)

5) IRNSS CDMA Ranging Stations (IRCDR)

6) Laser Ranging Stations

7) IRNSS Data Communication Network (IRDCN)

CDMA extending is being completed by the four IRCDR stations all the time for all the NavIC satellites. The INC built up at Byalalu performs remote tasks and information accumulation with all the ground stations. The IRNWT has been set up and is furnishing IRNSS framework time with an exactness of 2 ns $\left(2.0 \times 10^{-9} \mathrm{~s}\right)(2$ sigma $)$ with respect to UTC. 14 IRIMS are right now operational and are supporting NavIC operations. Laser extending is being completed with the help of ILRS stations the world over. Route Software is operational at INC since 1 August 2013. The IRDCN has built up earthly and VSAT connects between the ground stations. Seven $7.2 \mathrm{~m}$ FCA and two $11 \mathrm{~m}$ FMA of IRSCF is right now operational for LEOP and on-circle periods of IRNSS satellites [3]. All the route parameters viz. satellite ephemeris, clock revisions, trustworthiness parameters and auxiliary parameters viz. ionopostpone remedies, time counterbalances with respect to UTC and different GNSS, chronological registry, instant message and earth direction parameters are created and uplinked to the shuttle naturally [1].

Signal: Standard Positioning Service are modulated by a 1 MHz BPSK signal and a Precision Service will use BOC $(5,2)$ are the two NavIC signals consists of S1 band (2492.028 $\mathrm{MHz}$ ) and L5 (1176.45 MHz). An informing or data interface is implanted in the NavIC framework. The navigation signals themselves would be transmit in the $S$ band recurrence (2-4 $\mathrm{GHz}$ ) and communicate through a staged exhibit reception apparatus i.e., antenna to keep up required inclusion and signal quality. This element permits to send admonitions to a particular geographic region. For instance, fishermen utilizing the framework can be cautioned about a cyclone [3]. 


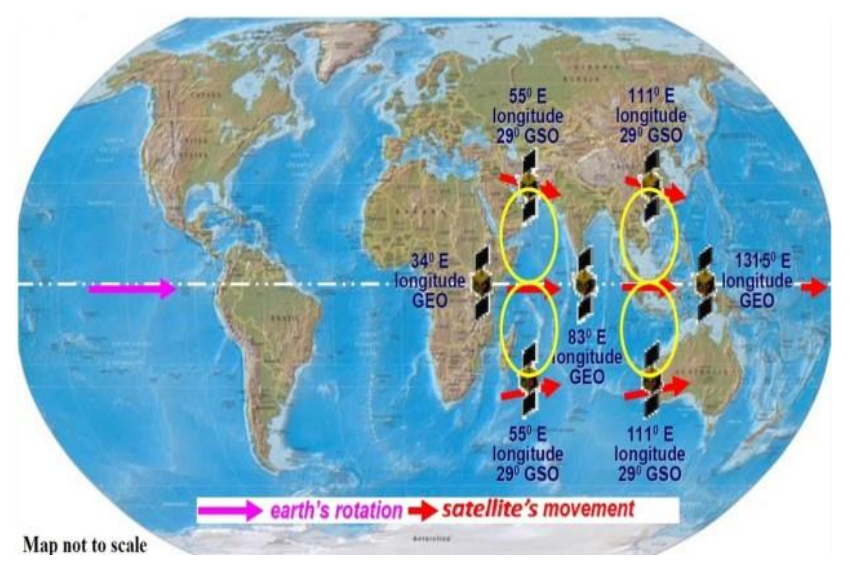

Fig. 1. NavIC/ IRNSS Constellation.

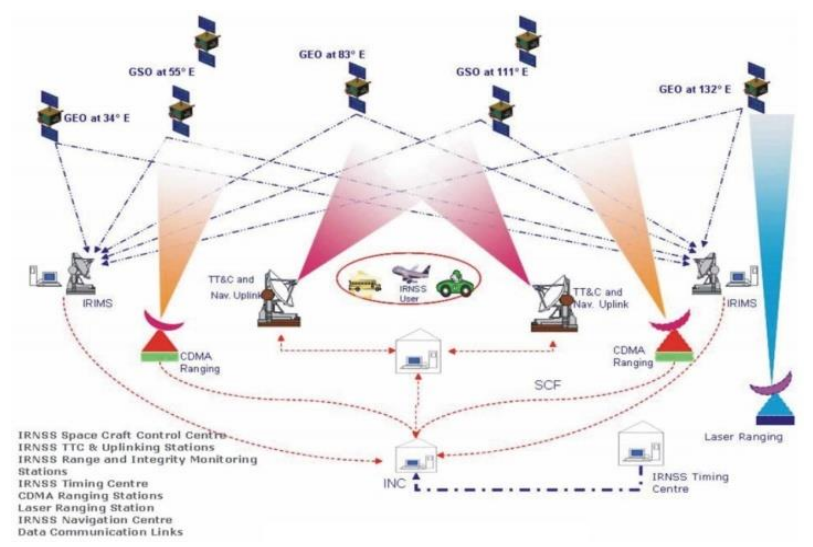

Fig. 2. IRNSS Operation Model [ISRO].

Accuracy: The framework is expected to give a complete position exactness of superior to anything 10 meters all through Indian landmass and superior to anything 20 meters in the Indian Ocean just as an area broadening roughly 1,500 km (930 mi) around India. NavIC has dual frequencies i.e., S1 and L5 bands whereas GPS which is dependent on L band only. At the point when low-recurrence sign goes through the climate, its speed changes because of air unsettling influences. For India's situation, the real delay is evaluated by estimating the distinct differences in two frequency i.e., $\mathrm{L}$ and $\mathrm{S}$ band. Thusly, NavIC isn't subject to any model to discover the recurrence mistake and is more precise or near to the GPS [6][8][10].

Major applications of NavIC are: Disaster Management, Terrestrial, Precise Timing, Aerial and Marine Navigation, Mapping, Vehicle tracking, Integration with mobile phones, Voice and visual navigation for drivers and fleet management, Terrestrial navigation aid for hikers and travelers, and Geodetic data capture.

There are various challenges involved in replacing the existing GPS navigation system with autonomous regional navigation system. In this paper aims at plotting or mapping the rover position in real time using both GPS and NavIC receivers. The purpose of real time mapping of rover position is to identify the geographical region with similarity and differences between GPS and NavIC navigational systems [7] [9].
This paper covers these contents in the following chapters: That is about the field survey carried out by the research team, Application software and Hardware development, Observation and Analysis, Conclusion and Future work.

\section{FIELD SURVEYS}

A total of five field surveys had been conducted. The survey had been conducted on a rover with IRNSS-UR, antenna and Laptop with IRNSS-UR application installed inside the vehicle shown in Fig. 3. IRNSS/ NavIC system at the user end identifying the following parameters are (a) the variations in experimental setup when the rover is on move at different speeds due various factors like vibrations and soon; (b) no vehicle movement trace / marker on GUI provided by ACCORD during its movement like Google navigation map.

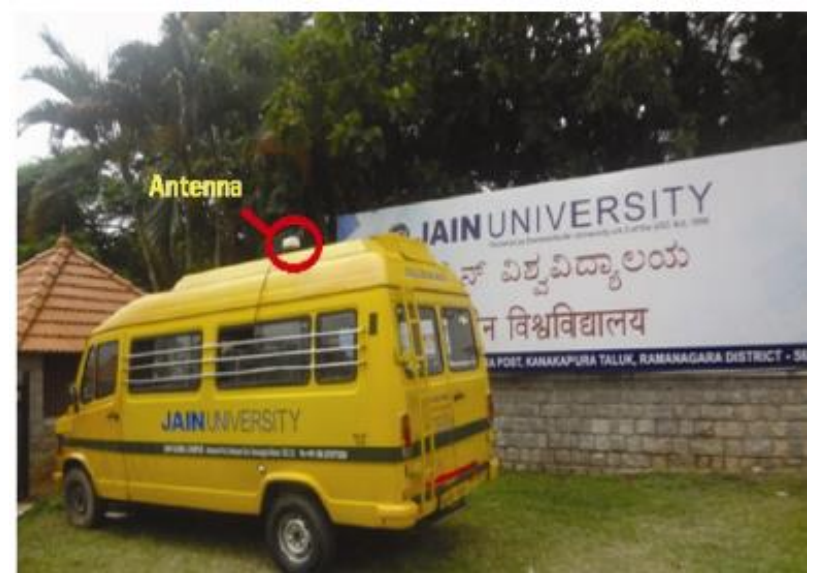

Fig. 3. Antenna Setup in Mobile Vehicle.

The hardware device which basically consists of Raspberry pi board and other peripherals have been developed an application in order to read NMEA (National Marine Engineering Association) data from the IRNSS-UR at $1 \mathrm{~Hz}$.

The application is programmed to read the NMEA from the IRNSS-UR receiver, parsed the NMEA data, storage and display colored marker on GUI to indicate the current vehicle position. The hardware and application has been tested during this survey and noted the bugs / issue / short comings of both hardware and software.

Few of the bugs encountered during this survey are:

1) The device was able to read data but the delay for data reading was incremental and that leads to incremental time differences between current time and time read by the device.

2) When there is switch between poor signals strength to good signal strength (vice-versa) the parsing of NMEA data was incorrect.

Two separate applications developed to fix the problem of -"NMEA data reading at incremental delay which causing loss of data".

1) One of the applications is meant for reading NMEA data, saving data in separate text file with auto count. The rate of creating the text file is fixed at the rate of every 30 seconds (new text file / $30 \mathrm{sec}$ ). 


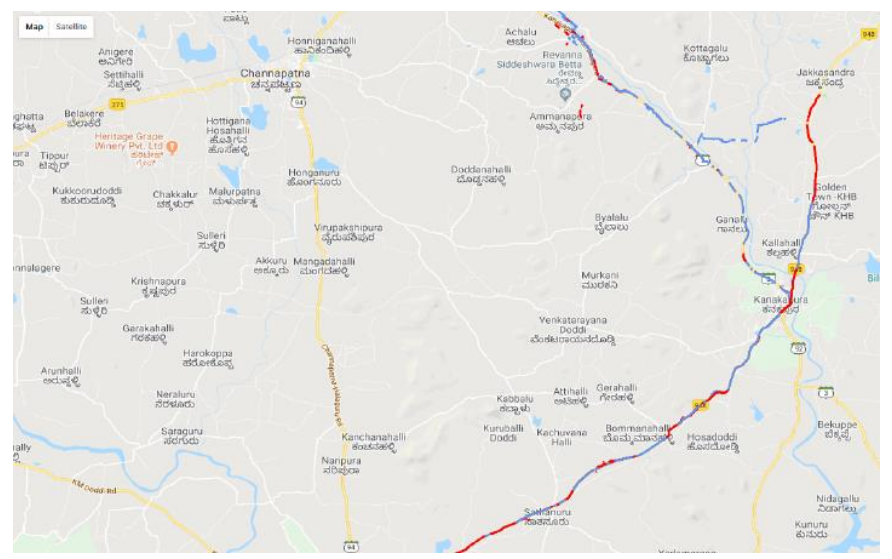

Fig. 4. Survey Data Plotted from NavIC and GPS.

2) The second application reads the NMEA data from these files and displays the details like date; time; lat; long; alt; positions of both IRNSS/ NavIC (blue color) and GPS (red color) separately on display shown in Fig. 4. The IRNSS and GPS traces are also displayed on the base maps to watch / trace the movement of vehicle in real time.

\section{ApPlication Software DeVElopment}

The NavIC receiver interface and to retrieve the real-time positional data (latitude, longitude, altitude) in the form of waypoints, satellite information like Number of Satellites, Relative accuracy, number of SVs in view, time zone.etc. The data retrieved is a set of values such that for every IRNSS value there is a corresponding GPS value. The data retrieved is then used to display the information on GUI like distance travelled, delta value of waypoints (difference in value w.r.t previous waypoint), the positional information, the satellite Information and also the waypoints for GPS and IRNSS are displayed on the basemap with regional boundaries. Then all of retrieved information is logged in files which allow the application to revisit the journey step by step as in real-time survey, this allows us to retest the software after any alteration without the need for an additional field survey. The positional data is stored in database for various reasons like the ability to derive important information from the field survey, to plot the positional data on the map, create reports and perform data analysis (Fig. 5).

READER1: The reader1.py file takes user input for date (ddmmyy) and creates a folder (if it does not exist already) for $\log$ files for that date (it is up to the user to use new directory or use existing directory) and starts writing the data received from the receiver into the log files, if the data is unavailable the receiver will still be continuously flushing empty NMEA format data and writing it to the log file. The data is written in a new $\log$ file by incrementing the logfile count every 30 s and if the receiver is disconnected it keeps waiting for the connection and continues from the previous point and if the application is restarted it can be made to continue from next $\log$ count.

MAIN1: The main1.py file again takes user input for the date (ddmmyy) and log count, checks if a folder exists and then starts reading the $\log$ files for that date from the given count (generally enter 1), this allows us to run the application for any survey data past or real time, if run in a real time session it reads all $\log$ files from the given log count and starts doing the following steps:

1) Parses NMEA data for GPGGA, IRGGA, GPRMC,GPGSA, GPGSV strings

2) Extracts Date, Time, Lat, Long, Alt for GPS and IRNSS

3) Inserts the extracted data into database

4) Displays the new information through the GUI

When running the application for a real-time session, reader1, py is started first and then main1, py, where as if user just wants to display previous journey data, directly main1.py is run by giving the date for the previous journey.

Database: This requires MySQldb middle to interface with MySQL database, we are all the coordinates from GPS and IRNSS separately and also a table to bookmark the locations. Each entry includes index, latitude, longitude and time.

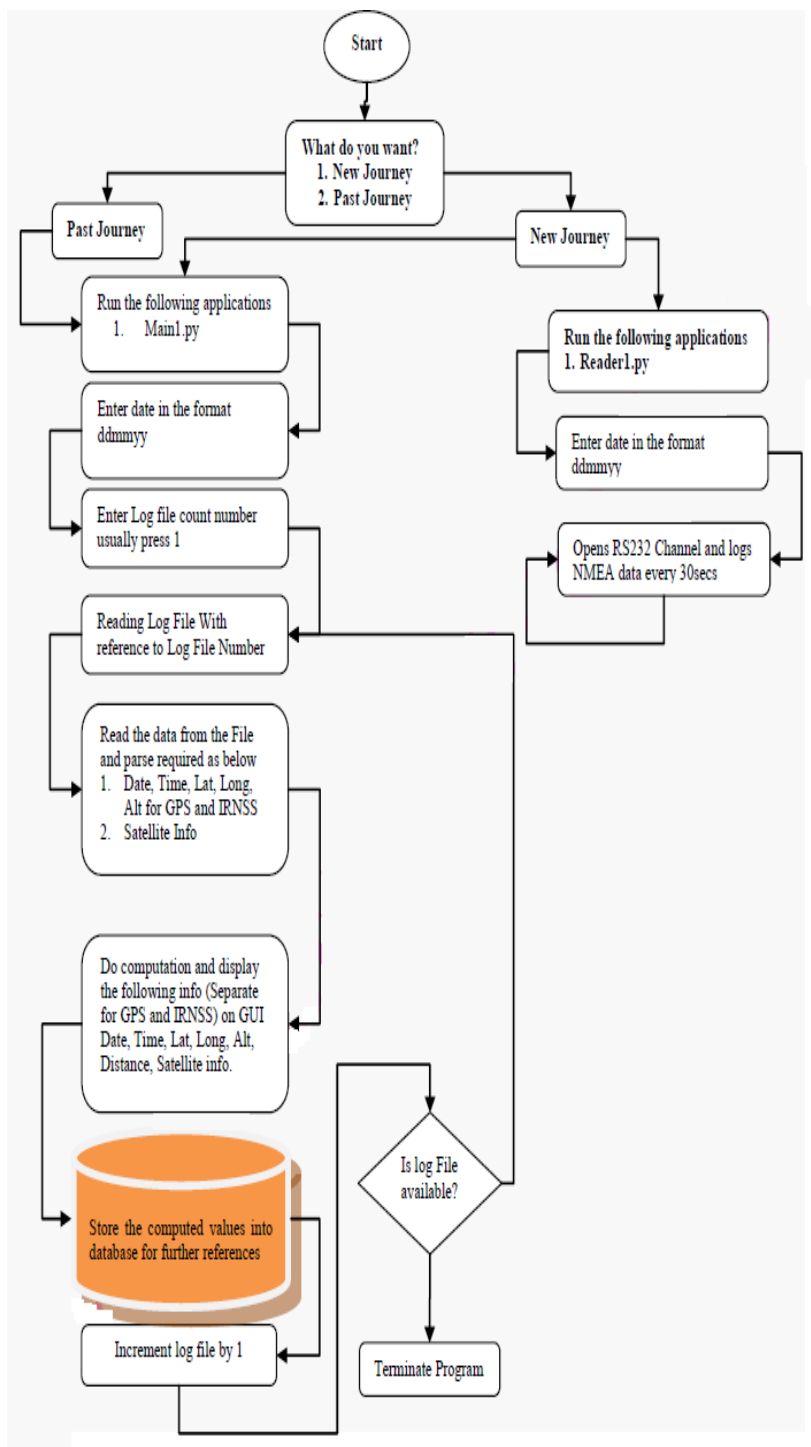

Fig. 5. Flow of the Process. 
\# SQL DATA BASE Host: Local ;UserName: faraz1 ; Pwd:ppp ; DB: temps db = MySQLdb.connect("localhost", "faraz1", "ppp", "temps") con=db.cursor() \# Inserting parsed IRNSS values into Database (Similar to GPS values) sql = "INSERT INTO II1 (ILON,ILAT,IALT,ITIME,DATE) VALUES('"+str(ilon[it])+"','"+str(ilat[it])+"','"+str(ialt[it])+"',' "+str(itime[it])+"',"+d1+");" con.execute(sql) db.commit() \# Inserting bookmarked GPS values into Database sql = "INSERT INTO gps_bookmark (NAME,LON,LAT,ALT) VALUES('"+str(name)+"','"+str(glon[0])+"','"+str(glat[0])+"',' "+str(galt[0])+"');" con.execute (sql) \#Finalizing and closing connection db.commit() db.close()

Graphical User interface: This includes loading the main Application interface, Map components and tool bar to interact with the Map. The GUI updates itself every few times in a second and allows reflecting any triggered changes. Basemap allows us to plot the Map with some features like country boundaries, State boundaries; terrain etc. city layout features can also be added.

Inbuilt modules: Time, sys and OS modules are inbuilt module they are used as follows. OS module is used for performing directory and file related operations like making new directories and checking if directory or file exists etc.

Text extraction and pattern matching: re-module is used for text extraction using regular expressions, helping us to extract strings matching certain pattern.

Haversine: This module is used to calculate distance between 2 points on earth taking into consideration the curvature of the earth since directly using the coordinates will not give cutest results.

\section{HARDWARE DEVELOPMENT}

Fig. 6 shows the block diagram representation which consists of the portable electronic device includes raspberry pi model B, 7 inch touch screen and USB-TTL-RS232 converter. The IRNSS-UR is interfaced with the designed portable electronic device through USB-TTL-RS232 converter. The IRNSS-UR receives the L1, L5 and S1 band signals from IRNSS and GPS satellites and provides NMEA data format at RS232 channel. The portable electronic device reads the received NMEA data and stores the data in the text file format at a frequency of every 30 seconds. The algorithms were developed to read the real time NMEA information from the text files and are parsed to get date, time, latitude, longitude, altitude, signal strength and satellite information. These parsed data are further processed to display the information on the display screen and also to trace the corresponding waypoints of GPS and IRNSS on base-map using two different colors. The algorithms are also responsible to save the parsed data into SQL database for further offline analysis. This portable electronic device integrated with developed algorithms helps us to trace the real time waypoints received from GPS receiver and IRNSS receiver. This helps us to map both GPS and IRNSS rover positional information and to indentify the coverage area or rover movement simultaneously in real time.

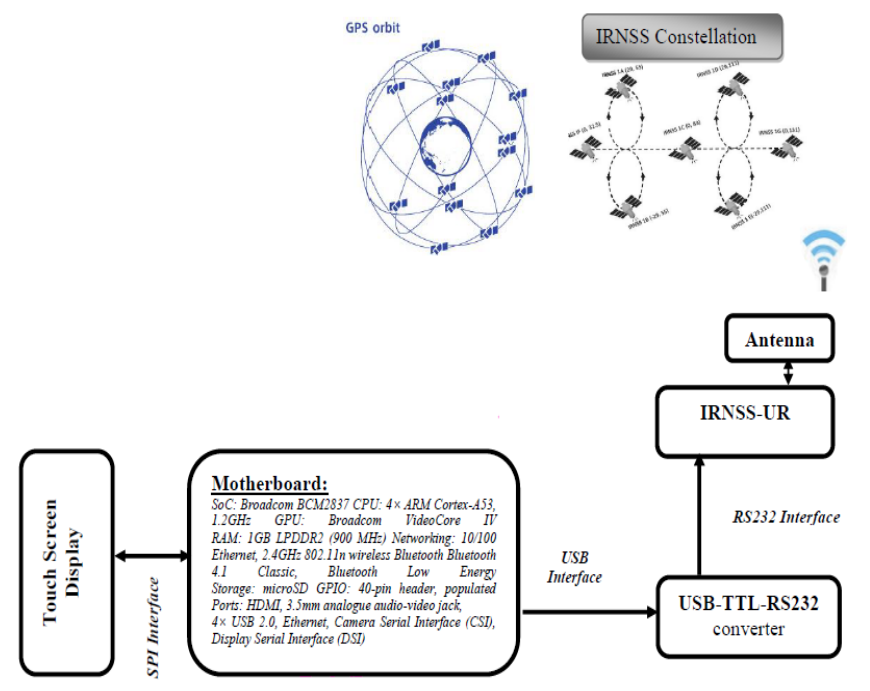

Fig. 6. Block Diagram Representation.

\section{A. Raspberry Pi}

The Raspberry $\mathrm{Pi}$ is a series of small single board computers developed in the UK by the Raspberry $\mathrm{Pi}$ Foundation. All models feature a Broadcom System on Chip (SoC) with an integrated ARM compatible CPU and On-chip Graphical Processing Unit (GPU). Processor speed ranges from $700 \mathrm{MHz}$ to $1.2 \mathrm{GHz}$ for the Pi 3 and on-board memory range from $256 \mathrm{MB}$ to $1 \mathrm{~GB}$ RAM. Secure Digital cards are used to store the operating system and program memory in either SDHC or Micro SDHC sizes. Depending on the model; the boards have either a single USB port or up to four USB ports. For video output, HDMI and composite video are supported, with a standard $3.5 \mathrm{~mm}$ phono jack for audio output. Lower level output is provided by a number of GPIO pins which support common protocols. The B-models have an 8P86 Ethernet port and the Pi 3 and Pi Zero W have on-board Wi-Fi 802.11n and Bluetooth. The default firmware is closed source, while an unofficial open source is available. The major components used in the raspberry Pi shown in Fig. 7.

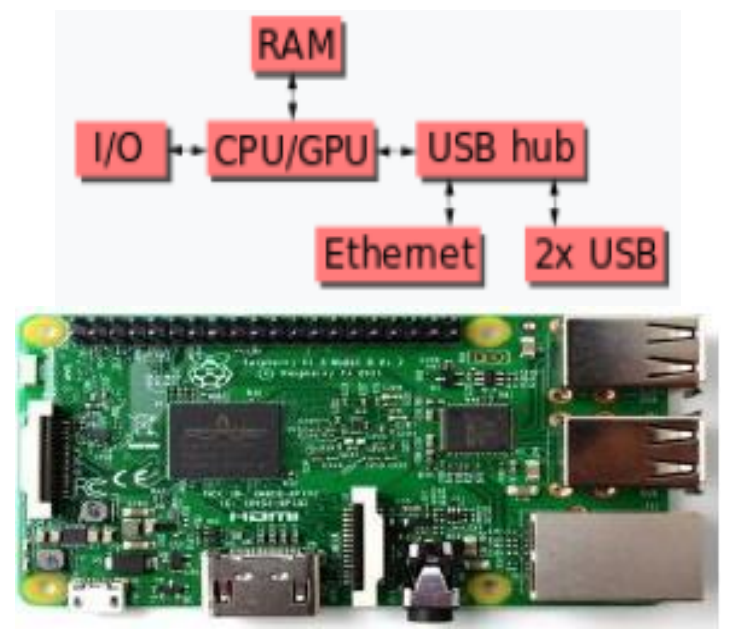

Fig. 7. Components of Raspberry Pi and Model. 
The Ethernet adapter is internally connected to an additional USB port. In Model $\mathrm{A}, \mathrm{A}+$, and the Pi Zero, the USB port is connected directly to the System on Chip (SoC). On the Pi 1 Model B+ and later models the USB/Ethernet chip contains a five-point USB hub, of which four ports are available, while the Pi 1 Model B only provides two. On the Pi Zero, the USB port is also connected directly to the SoC, but it uses a micro USB (OTG) port.

A typical block diagram of the IRNSS-UR is shown in Fig. 8. IRNSS-UR is expected to receive, down convert and demodulate the transmitted satellite signals both at L5 $(1176.45 \mathrm{MHz})$ and S1 (2492.028 MHz) band frequencies. Most importantly, IRNSS-UR generates measurements precisely with respect to the external/internal trigger such as 1 Pulse per Second (PPS). In addition, IRNSS-UR shall also include capability to process Global Positioning System (GPS) L1 Coarse/Acquisition (C/A) signals centered at $1575.42 \mathrm{MHz}$ and generate measurements with respect to the external/internal trigger. The receiver shall output the user position computed using L5 only, S1 only; L1 only, combined L5 and GPS and combined S1 and GPS and combined IRNSS and GPS. Control inputs to the system include $10 \mathrm{MHz}$ external reference clock, 1-PPS signal and commands/data through Ethernet and/or RS232 interface. The IRNSS-UR shall also have an USB port for IF sampled data collection into the PC and an external storage device.

\section{B. Antenna Setup}

Antenna module contains a mounting interface and an RF connector on bottom plate. Mounting interface is a standard M16 nut and can be mounted on standard M16 pipe/threaded pole (Fig. 9) [4]. Ensure there are no metal parts nearby the antenna for optimistic performance during installation. RF connector is a TNC Female type, through which the RF signal received by the antenna is fed to the receiver and the DC supply from the receiver is fed to the antenna LNA.

1) The antenna mounting site should provide full 360degree visibility of the horizon. Any physical obstruction having an apex that makes an angle more than 5 degrees with the antenna phase centre, degrades the unit performance by blocking the satellite signals.

2) Ensure that there is no metal objects/plates touching or very close to the antenna. This is because the metal object alters the gain pattern of the antenna.

\section{Receiver Setup}

The back panel of the IRNSS-UR is as shown in the Fig. 10(a). The details and the functionalities of the ports in the back panel of the IRNSS-UR are as mentioned in Table I and the front panel of the IRNSS-UR is as shown in Fig. 10(b). The details and the functionalities of the ports in the front panel of the IRNSS-UR are as mentioned in Table II.

The steps to be followed depending on the Users' data requirement in a particular signal/data output are (Fig. 11): a) To view the NMEA data in the GUI: Connect RS 232 cable from Laptop to the NMEA/LCD port of receiver through the USB-to RS-232 converter (Table III).

b) To collect IF samples into PC: Connect USB cables from Laptop to the IF SAMPLES TO PC port and SD Card Data TO PC port of receiver.

c) To collect IF samples into Hard-disk: Connect USB cable from External Hard disk to the IF SAMPLES TO HARD DISK port of receiver.

d) To run the Receiver with External clock source: For External clock source, connect SMA Cable from external clock source to the $10 \mathrm{MHz}$ IN port of receiver.

e) To Latch the Receiver measurements with external PPS: Connect SMA Cable from external PPS source to the EXT PPS IN port of receiver.

f) To log the Navigation data of IRNSS into SD card: Insert SD card into the SD CARD slot of receiver.

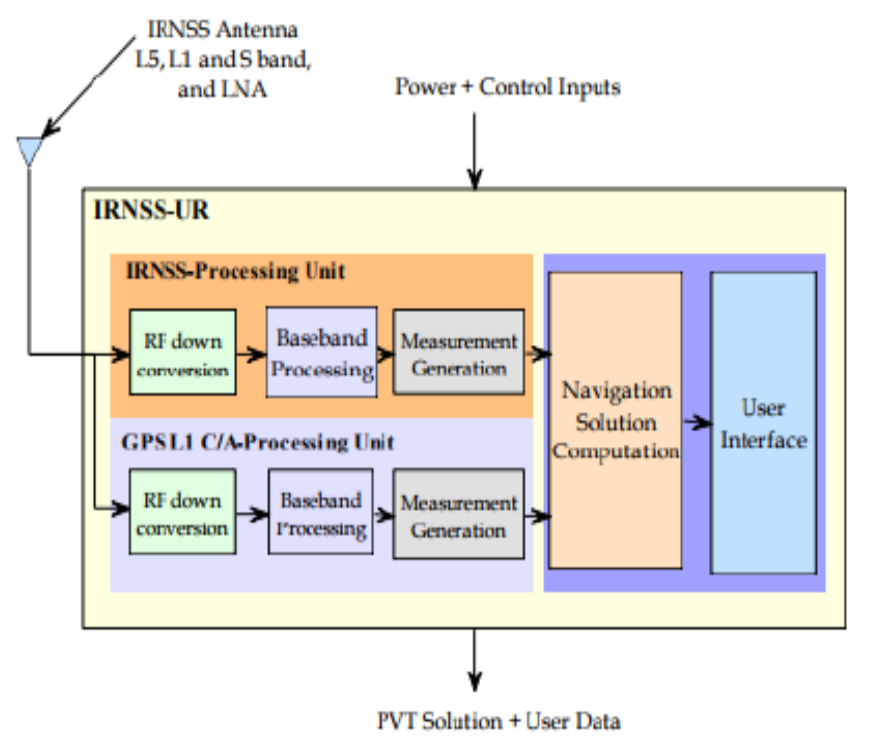

Fig. 8. High Level Block Diagram of IRNSS-UR.

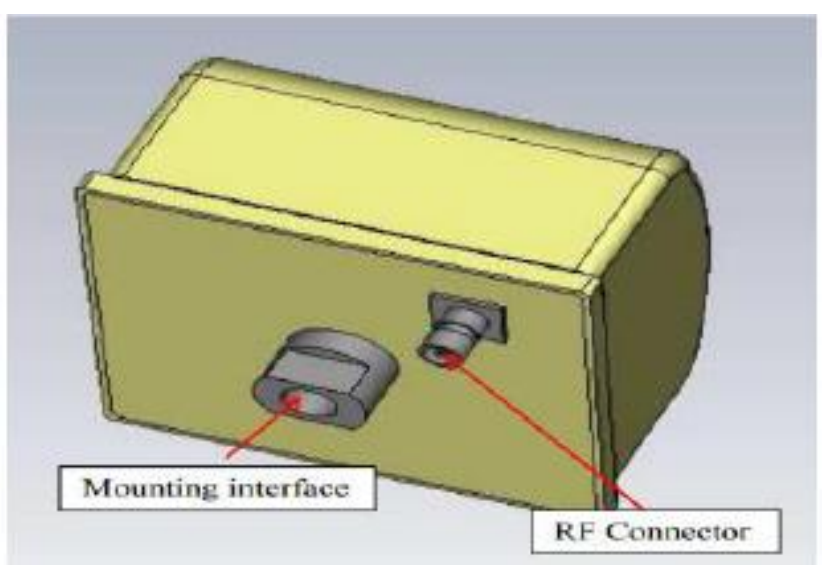

Fig. 9. IRNSS Antenna. 


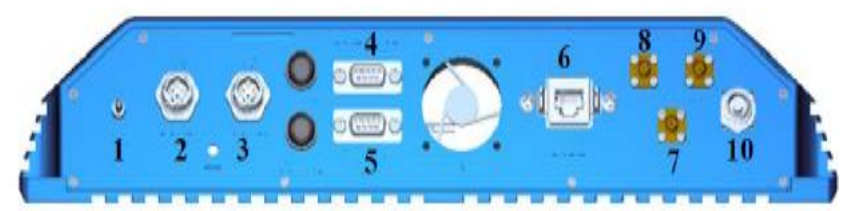

(a) Receiver Back Panel Showing Pins.

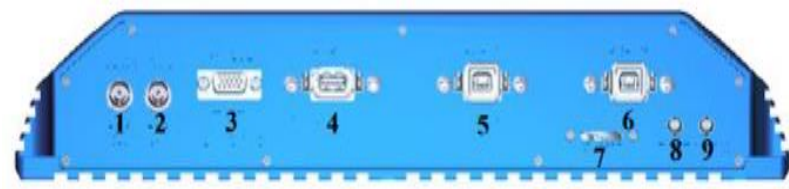

Fig. 10. (b) Receiver Front Panel Pins.

TABLE. I. RECEIVER BACK PANel Pins AND THEIR FunCtionalities

\begin{tabular}{|c|c|c|}
\hline Sl. No. & Ports & Functionality \\
\hline 1 & ON/OFF & $\begin{array}{l}\text { Gives control to power ON/OFF the IRNSS- } \\
\text { UR }\end{array}$ \\
\hline 2 & $12 \mathrm{~V}$ DC IN & $12 \mathrm{~V} \mathrm{DC}$ supply is fed from battery \\
\hline 3 & $230 \mathrm{~V}$ AC IN & $230 \mathrm{~V}$ AC supply is fed from mains \\
\hline 4 & NMEA/LCD & $\begin{array}{l}\text { Used for NMEA data output, Ethernet IP } \\
\text { configuration, and also for data } \\
\text { communication with the detachable LCD (in } \\
\text { the field environment) }\end{array}$ \\
\hline 5 & SERVICE PORT & Factory Maintenance Port \\
\hline 6 & $\begin{array}{l}\text { ETHERNET } \\
\text { PORT }\end{array}$ & $\begin{array}{l}\text { To display status on Graphical User Interface } \\
\text { running on host computer and also to receive } \\
\text { commands }\end{array}$ \\
\hline 7 & PPS IN & $\begin{array}{l}\text { External PPS is fed through this port for } \\
\text { measurement latching }\end{array}$ \\
\hline 8 & $10 \mathrm{MHz}$ IN & $\begin{array}{l}\text { External } 10 \mathrm{MHz} \text { clock reference is fed } \\
\text { through this port }\end{array}$ \\
\hline 9 & $10 \mathrm{MHz}$ OUT & $\begin{array}{l}\text { Receiver gives out } 20 \mathrm{MHz} \text { clock reference } \\
\text { through this port. }\end{array}$ \\
\hline 10 & ANT & $\begin{array}{l}\text { IRNSS L5, S and CPS L1 RF signals are fed } \\
\text { through this port }\end{array}$ \\
\hline
\end{tabular}

TABLE. II. RECEIVER Front PANEL PINS AND THEIR FUNCTIONALITIES

\begin{tabular}{|l|l|l|}
\hline Sl. No. & Ports & Functionality \\
\hline 1 & IRNSS PPS OUT & IRNSS 1 PPS reference output \\
\hline 2 & GPS PPS OUT & GPS 1 PPS reference output \\
\hline 3 & RS 232 Monitoring & $\begin{array}{l}\text { PRN code, PRN code epoch, bit synch, data } \\
\text { and data clock are given outside through } \\
\text { this port }\end{array}$ \\
\hline 4 & $\begin{array}{l}\text { IF samples to Hard } \\
\text { disk }\end{array}$ & $\begin{array}{l}\text { Intermediate frequency samples are } \\
\text { transmitted to the external hard disk through } \\
\text { this USB port }\end{array}$ \\
\hline 5 & $\begin{array}{l}\text { SD Card Data to } \\
\text { PC }\end{array}$ & $\begin{array}{l}\text { Stored 25 bps navigation data is transmitted } \\
\text { to the host computer through this USB port }\end{array}$ \\
\hline 6 & IF samples to PC & $\begin{array}{l}\text { Intermediate frequency samples are } \\
\text { transmitted to the host computer through } \\
\text { this USB port }\end{array}$ \\
\hline 8 & SD Card & $\begin{array}{l}\text { 25 bps navigation data is stored in the SD } \\
\text { card }\end{array}$ \\
\hline 9 & MATT & $\begin{array}{l}\text { Indicates 12 V DC is connected to the } \\
\text { IRNSS - UR }\end{array}$ \\
\hline
\end{tabular}

TABLE. III. LIST OF CABLES

\begin{tabular}{|l|l|}
\hline Cable Name & Quantity \\
\hline 230 V AC cable & 1 \\
\hline 12 V DC Battery cable & 1 \\
\hline RS 232 cable (1m) for NMEA port & 1 \\
\hline LCD cable (1 feet) & 1 \\
\hline Ethernet cable & 1 \\
\hline LMR 400 DB antenna cable & 1 \\
\hline USB type B to type A cable & 2 \\
\hline Battery charger adapter and cable & 1 -set \\
\hline USB to RS-232 converter & 1 \\
\hline
\end{tabular}

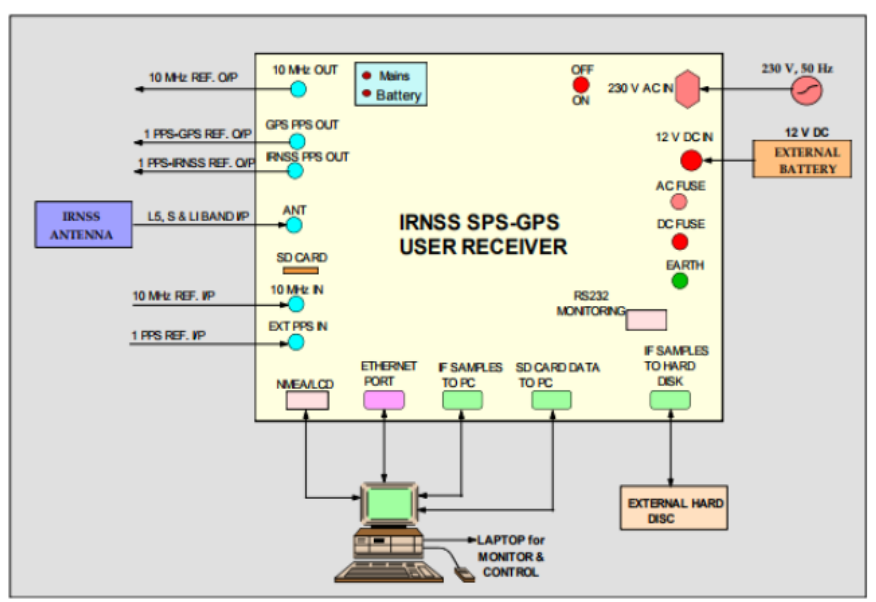

Fig. 11. Interconnection Diagram of IRNSS -UR.

\section{Operating Systems}

The Raspberry Pi Foundation recommends the use of Raspbian, a Debian-based Linux operating system which includes Ubuntu MATE, Snappy Ubuntu Core, Windows 10 IoT Core, and RISC OS.

To set up a blank SD card with NOOBS:

1) Format an SD card which is 8GB or larger as FAT.

2) Download and extract the files from the NOOBS zip file.

3) Copy the extracted files onto the SD card that you just formatted, so that this file is at the root directory of the SD card. Please note that in some cases it may extract the files into a folder; if this is the case, and then copy across the files from inside the folder rather than the folder itself.

4) On first boot, the "RECOVERY" FAT partition will be automatically resized to a minimum, and a list of OSes that are available to install will be displayed.

\section{E. Hardware Packaging}

3D Packaging: The below pictures represents the 3D design of Back, Front and Battery panels. The complete device is enclosed in these three panels. The complete product packaging is designed such that the device is portable and battery operated. Fig. 12 shows the display model along with 3D printing enclosure. 


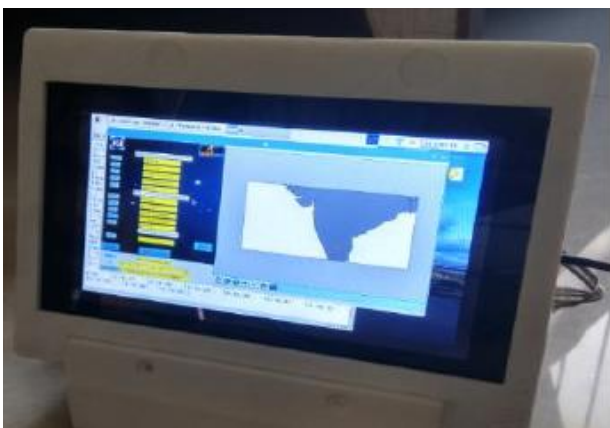

Fig. 12. Packed Display with Results.

\section{OBSERVATION AND ANALYSIS}

The application software running on the hardware during the field survey test is

Step 1: The user need to run the Main1.py application and application waits for the user to enter the date in the ddmmyyyy format (Fig. 13).

Step 2: After entering the date in the ddmmyyyy format (Fig. 14). The Graphical User Interface that gives the GPS and IRNSS receiver Positional information with date and time separately. The GUI is also integrated with basemap on which the waypoints are traced with red and green marker and finally click on start journey button (Fig. 14).

Step 3: After clicking on Start Journey button. The user needs to enter the count for the log file in the terminal window. After every 30 seconds the log files are created containing the NMEA data of both GPS and IRNSS receiver (Fig. 15). The waypoints are also displayed on the base maps.

The positional information of a rover with respective to GPS and IRNSS is displayed as shown in the Fig. 16. The parameter Date, Time, Latitude, Longitude and Altitude are displayed.

The Base map displaying waypoints in Red (IRNSS) and Green (GPS) color are traced to show the rover movement (Fig. 17). The distance travelled by the rover is also calculated using haversine expression and is displayed at the left bottom corner of the GUI. The base map can be zoom in and out, and can be moved.

The Rover distance travelled with respective to the IRNSS and GPS are determined separately and displayed on the GUI (Fig. 18). The satellite information like the number of satellite visible and the delta distance are displayed.

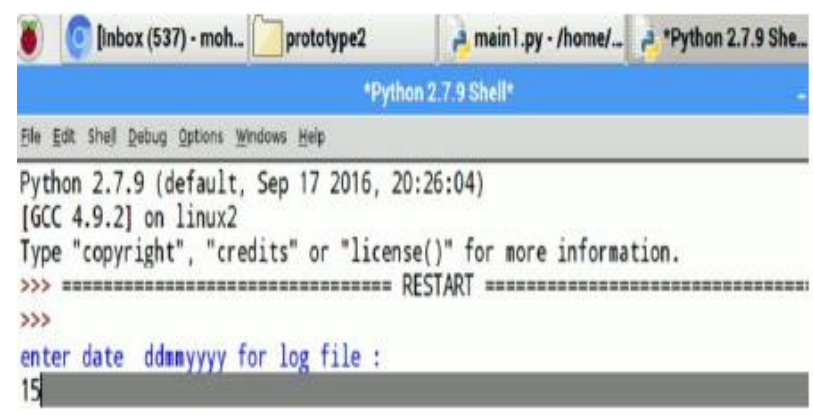

Fig. 13. Main1.py Application.

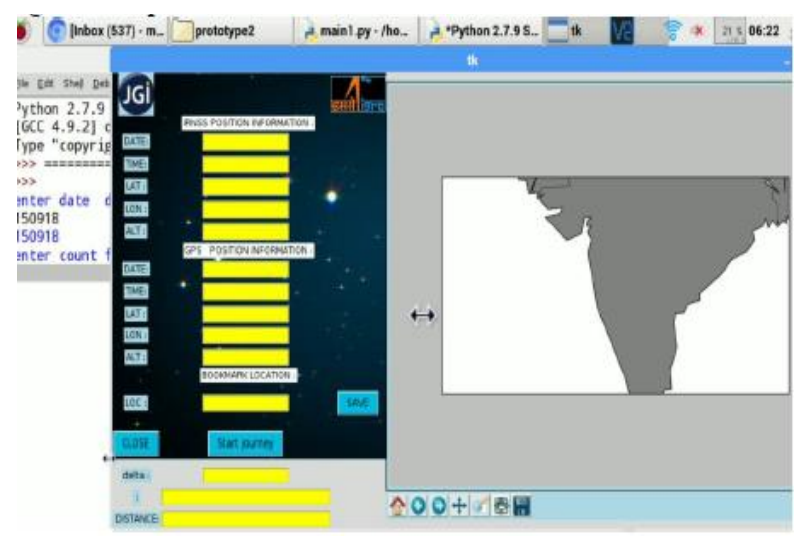

Fig. 14. Graphical user Interface.

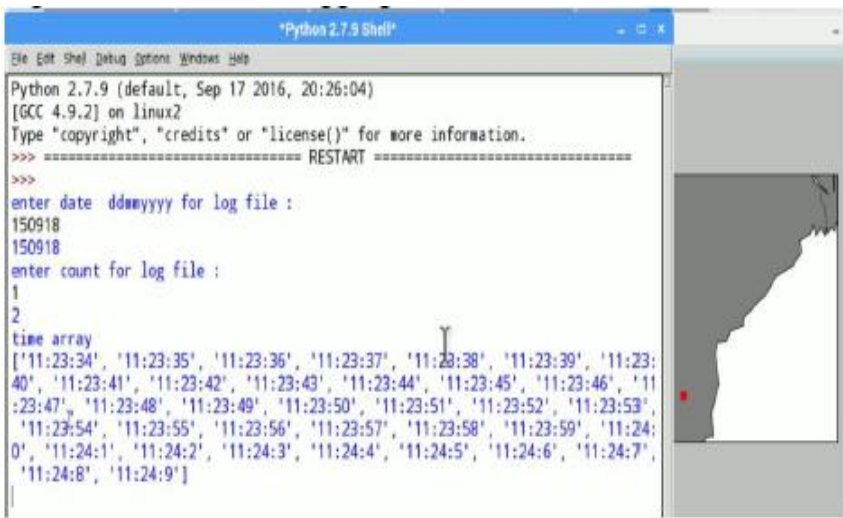

Fig. 15. NMEA Data Logging.

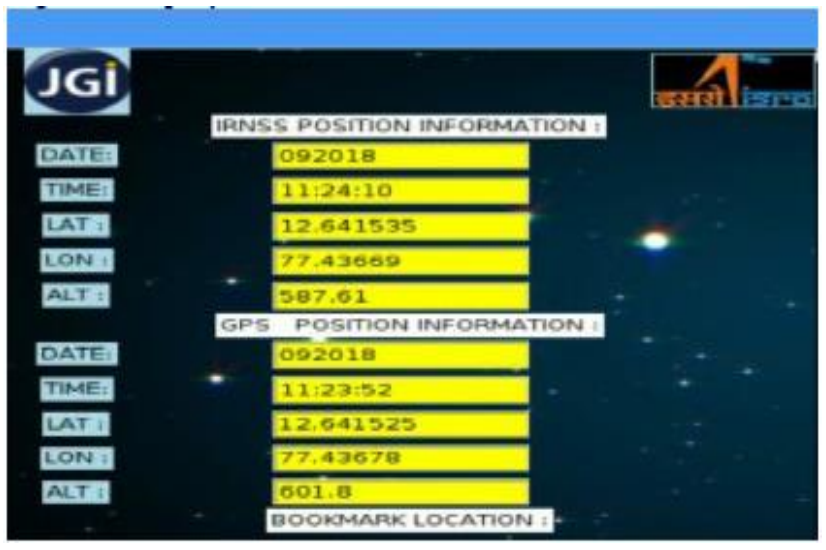

Fig. 16. Display Rover Positional Information.

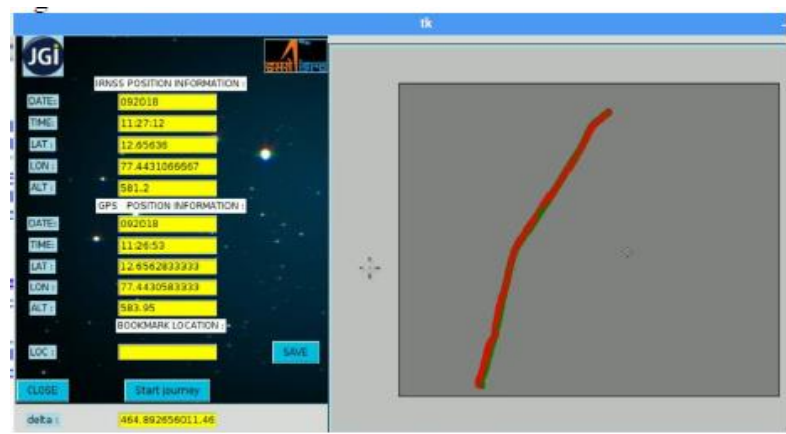

Fig. 17. Rover Movement. 


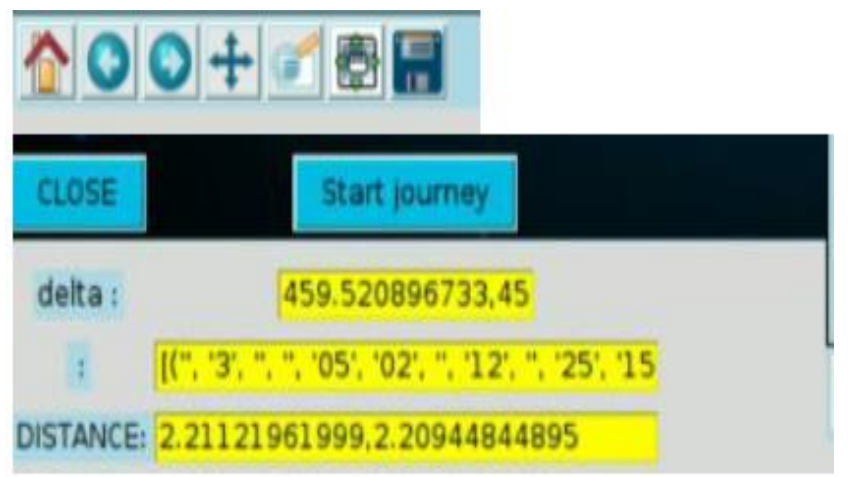

Fig. 18. Rover Distance Travelled.

The waypoints i.e., latitude and longitude of both GPS and IRNSS are loaded into Google maps to trace the rover movement using device logged data and accord logged data shown in Fig. 18.

Fig. 19 shows the GPS waypoints from the NavIC user receiver depicted by blue color markers, the journey shows a more consistent waypoints dataset than the previous datasets although there is small discontinuity only due to the obstructions like flyovers and other tall buildings or due to signal loss, the GPS data received by the portable device is compared with the above image to highlight irregularities and bottlenecks associated with the portable device or the code. The journey was made from Jain University located near Jakkasandra and the stopping point was near JP Nagar. The data stayed consistent despite the speed changes and the waypoints correctly coincided with the roads marked on the map, we can infer from this that there was no error due to shift of values as observed in the earlier surveys from the portable device readings. It can also be observed in the image that there were no outliers in data that were observed in IRNSS readings from the NavIC user receiver.

Fig. 20 shows the IRNSS waypoints from the NavIC user receiver depicted by red color markers, the journey shows a more consistent waypoints dataset than the previous datasets which is also observed in the GPS data above, although there is small discontinuity due to the obstructions like flyovers and other tall buildings or due to signal loss and also we can observe few outliers that are clearly result of a minor fault since the vehicle wouldn't have travelled to the points depicted by the outliers, the IRNSS data received by the portable device is compared with the above image to highlight irregularities and bottlenecks associated with the portable device or the code. This image shows the journey data made from Jain University located near Jakkasandra to the stopping point that was near JP Nagar. As with the GPS data, the IRNSS data also stayed consistent despite the speed changes and the waypoints correctly coincided with the roads marked on the map and also with GPS data from the NavIC receiver, we can infer from this that there was no error due to shift of values as observed in the earlier surveys from the portable device readings.

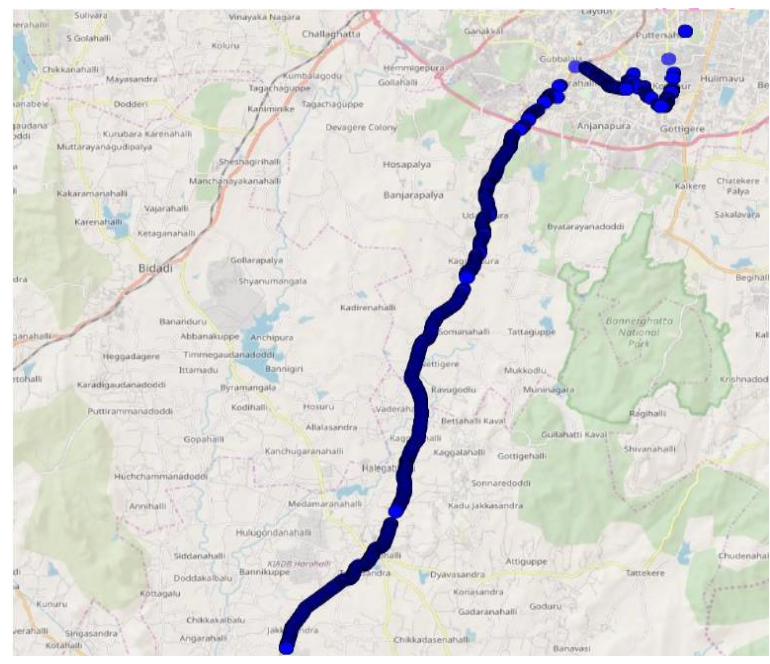

Fig. 19. Google Map using GPS Waypoints from Accord Logged Data.

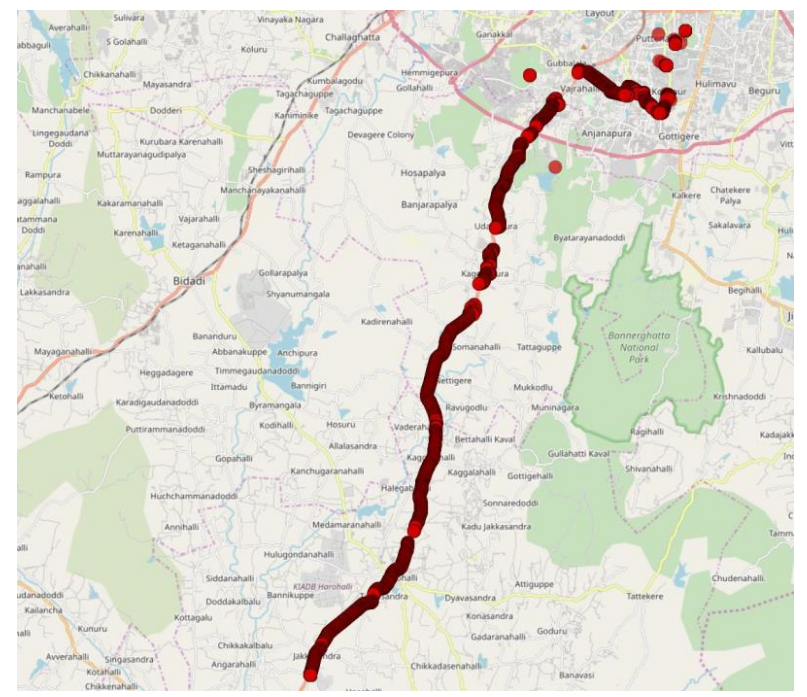

Fig. 20. Google Map using IRNSS Waypoints from Accord Logged Data.

\section{CONCLUSION AND FUTURE WORK}

As with the IRNSS data from the NavIC user receiver, the portable device IRNSS data also stayed consistent despite the speed changes and the waypoints correctly coincided with the roads marked on the map and also with GPS data from the NavIC receiver, we can infer from this that there was no error due to shift of values as observed in the earlier surveys from the same device and the device data quality was comparable to that of the Receiver data. One important observation is that the data is a slightly sparser than the original data, this is due to the fact that code reduces the frequency of taking the way points to avoid redundancy of data points. This device can also be used in mapping and surveying project. By utilizing these devices capabilities in surveying can potentially save companies cost and time. This will allow surveying positions in the shortest time possible. Mapping can be done in case of highways, farmlands, rivers, power lines, etc. 
This device can be further developed to guide and monitor the all transportation vehicles including aircrafts and keep them informed about the routes of the neighboring aircrafts in the airspace.

\section{ACKNOWLEDGMENT}

This research work was carried out by the grant sanctioned by ISRO File No. NGP22. The authors like to acknowledge the support of Space Applications Centre - Indian Space Research Organization (SAC-ISRO) by providing IRNSS receiver and encouraging us to work on this navigation studies. Authors thank Dr. G. Raju \& JAIN (Deemed-to-be University), Bangalore for the support to complete this work successfully. Scholar also acknowledges the Directorate of Minorities, Government of Karnataka for their Ph.D. Fellowship.

\section{REFERENCES}

[1] Chandrasekar, M. V. et al., Modernized IRNSS broadcast ephemeris parameters. J. Control Theory Inf., vol. 5, Iss.2, 2015.

[2] ISRO, Indian Regional Navigation Satellite System Signal in Space ICD for Standard Positioning Service (Version 1.0, ISRO-IRNSSICD- SPS1.0), Indian Space Research Organization, 2014.
[3] Mohmad Umair Bagali, Dr. Thangadurai N, "Embedded Board Development Interfaced with GPS/IRNSS/NavIC Receiver for Disaster Applications", Proc. Of SSRN, International Conference on Sustainable Computing in Science, Technology \& Management, pp. 416 - 426, Jaipur, 2019.

[4] Mohmad Umair Bagali, Naveen Kumar Reddy, Ryan Dias, Dr. Thangadurai N. The Positioning and Navigation System on Latitude and Longitude Map using IRNSS User Receiver. IEEE International Conference on Advanced Communication Control and Computing Technologies, Ramanathapuram, pp.122-127, 2016.

[5] Rao, V. G., Lachapelle, G. and Vijay Kumar, S. B., Analysis of IRNSS over Indian Subcontinent. J. Inst. Navigation, San Diego, 2011.

[6] Kaplan, "Understanding GPS: principles and applications", 2nd Ed, Artech House, 2006.

[7] Ganeshan, A.S., Rathnakara S.C., Gupta R., and Jain, A.K., Indian Regional Navigation Satellite System (IRNSS) Concept, ISRO Satellite Center Journal of Spacecraft Technology, 2005, 15(2), pp. 19-23.

[8] Mohmad Umair Bagali, Thangadurai N, "Application Specific Embedded Board Development Interfaced with GPS/IRNSS Receiver for Environmental Monitoring", International Journal of Innovative Technology and Exploring Engineering, Vol.8, Iss.8, pp. 26282637,2019.

[9] Grewal, M.S., Weill, L.R., and Andrews, A.P., Global Positioning Systems, Inertial Navigation and Integration, Wiley Publications, 2007, 2nd edition.

[10] Van Diggelen, F., GNSS Accuracy: Lies, Damn Lies, and Statistics, GPS world, 2007, pp. 26-32. 